\title{
Robotic perineal radical prostatectomy with high prostate volume
}

\author{
Volkan Tugcu, Abdulmuttalip Simsek, İsmail Yigitbasi, Mustafa Gürkan Yenice, Selcuk Sahin, \\ Ali İshsan Tasci
}

Bakirkoy Dr. Sadi Konuk Research and Training Hospital, Department of Urology, Istanbul, Turkey.

\begin{abstract}
Summary Background: Minimally invasive techniques are ever improving and are preferred more.

Many techniques were developed in radical prostatectomy operations. Robotic radical prostatectomy with the perineal approach is a new technique.

Case presentation: A 66-year-old male patient presented because of lower urinary tract symptoms, a PSA value of 5.5 $\mathrm{ng} / \mathrm{ml}$ was detected, prostate biopsy was performed under transrectal ultrasound guide, a Gleason 3+3 adenocarcinoma on 3/12 foci was reported at pathology. Robotic perineal radical prostatectomy ( $r$-PRP) operation was performed in the patient who had a prostate volume of $130 \mathrm{cc}$ with middle lobe and a body mass index of 32 without additional disease. The duration of operation was 140 minutes in total and the duration at the console was 95 minutes, the amount of bleeding was $85 \mathrm{cc}$ and no intraoperative complication was detected.

Conclusion: $r$-PRP is a technique that can be applied safely without prolonging the operation period and without additional morbidity to the patient, preserving the oncologic and functional outcomes in patients with surgical history and large prostate volume.
\end{abstract}

KEY WORDS: Robotic perineal radical prostatectomy; High prostate volume.

Submitted 15 January 2018; 20 January 2018

\section{INTRODUCTION AND BACKGROUND}

Since description of radical prostatectomy technique which has high morbidity and mortality, many methods have been used up to that time and the results have been presented. With the acquisition experience, the morbidity and mortality are reduced in parallel with the development of the technology and these operations can be performed safely in experienced centers with minimally invasive techniques. Despite the introduction of many new methods, some factors of the disease may cause this surgical technique to change, to apply another surgical technique or to give up the surgeon. One of these factors is the size of the prostate and when applied with different methods, prostate size can affect the duration of operation, amount of bleeding, postoperative urinary incontinence and erectile dysfunction (1). When open radical prostatectomy (ORP) compared to other techniques, it has been reported that the amount of bleeding is significantly increased as a negative factor (2). According to the data of the literature, laparoscopic radical prostatectomy (LRP) and robotic radical prostatectomy

(RRP) showed prolonged operation time and hospitalization (3). Since each technique has its own difficulties and limitations, it is only when the factors of the prostate do not affect the operation and when the comorbidities of the patient are also taken into account, the situation becomes more difficult and the development of new techniques will be inevitable. Robotic perineal radical prostatectomy (r-PRP) was developed and applied by Tugcu et al to 15 patients, indicated that this technique can be safely applied in centers with advanced robotic surgery experience (4). This technique with the perineal approach, is applied to a narrow surgical field without incision of endopelvic fascia and without abdomen involvement. When evaluating the surgical steps, a question comes to mind whether this technique is applicable to large prostates. In this case report, we aimed to prove that this technique can be safely applied to prostates with a large volume.

\section{Presentation of case}

A 66-year-old male patient presented because of lower urinary tract symptoms, a PSA value of $5.5 \mathrm{ng} / \mathrm{ml}$ was detected, prostate biopsy was performed under transrectal ultrasound guide and a Gleason 3+3 adenocarcinoma on 3/12 foci was reported at pathology.

At multiparametric magnetic resonance imaging (Figure 1a) of the patient, who had a laparotomy history due to ileus (Figure 1b), there was no extraprostatic spread when the PIRADS 3 lesion was detected, Radical-PRP

Figure 1a.

Screening middle lobe.

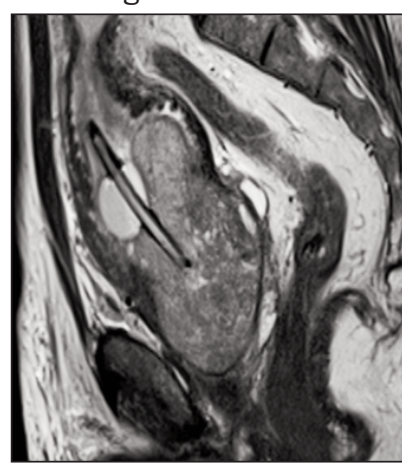

Figure 1b.

Laparotomy incision due to ileus.

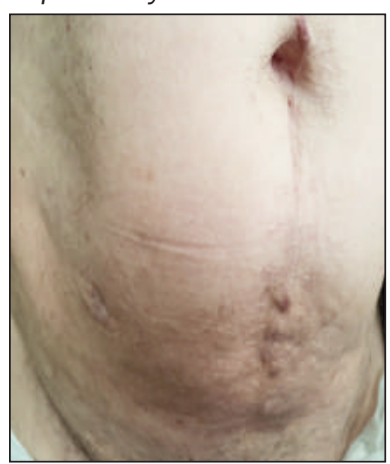


operation was performed in the patient who had a prostate volume of $130 \mathrm{cc}$ with middle lobe and a body mass index of 32 without additional disease. The duration of operation was 140 minutes in total and the duration of the console was 95 minutes, the amount of bleeding was $85 \mathrm{cc}$ and no intraoperative complication was detected. The drainage catheter and urethral catheter of the patient with no postoperative complication were removed on $2^{\text {nd }}$ and $7^{\text {th }}$ postoperative day, respectively, and the patient was discharged the same day of catheter removal.The pathology score was Gleason $3+3$ adenocarcinoma and the surgical margin was negative.

\section{Surgical technique}

The patient is taken to the exaggerated lithotomy position with 15 degrees of Trendelenburg. A urethral catheter is placed and the bladder is emptied. A sterile glove is placed in the rectum and the sides of the glove are stitched to the perineal skin. Thus, we aim to avoid rectum damage by using digital rectal examination during perineal dissections. A $6 \mathrm{~cm}$ semilunar incision is bilaterally made between tubercula ischiadica. The perineal dissection is terminated when the dissection margin reaches to the membranous urethra and the apex of the prostate is seen. Subcutaneous tissue laying under the incision borders is dissected deeply over the superficial perineal fascia to place the GelPOINT ${ }^{\circledR}$ (Applied Medical, Rancho Santa Margarita, CA, USA). Once the robotic system is docked (Figure 2), dissection is started from prostate apex and extended in to the lateral sides of the prostate and then deepened inferiorly to reveal the Denonvilliers' fascia covering the seminal vesicle compartment. Once the Denonvilliers' fascia is incised, vasa deferentes are bilaterally revealed, dissected and cut. Seminal vesicles are completely dissected and revealed. Then the membranous urethra is dissected and cut. The lateral prostatic pedicles are dissected and controlled using Hem-o-Lock ${ }^{\circledR}$ clips. After completing the lateral dissections of prostate bilaterally, the bladder neck is identified and incised with monopolar scissors, sparing and leaving intact the dorsal vein complex. Dissection was extended towards the bladder neck. Anterior bladder neck was incised using monopolar scissors. After dissection the bladder neck, the middle lobe of the prostate was observed and a vicryl suture was placed for traction of the middle lobe of the prostate (Figure 3a). With proper resection margin, posterior border of the bladder neck was cut and prostate fully dissected from the bladder (Figure 3b). Two of 4/0 V-Loc ${ }^{\mathrm{TM}}$ (Covidien, Mansfield, MA, USA)

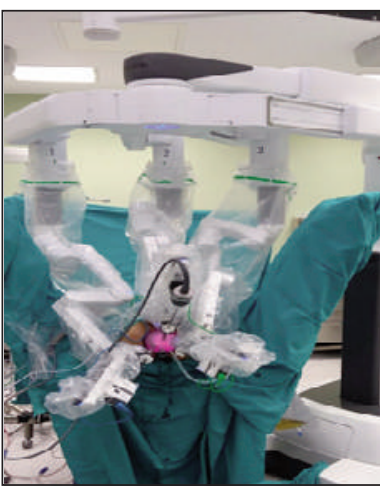
sutures are used in a running fashion starting from the Retzius side to rectal side of the bladder neck. The first suture is started at 12 o'clock on the bladder neck from outside to inside and then continued the

\section{Figure 2.}

Docking of the robotic system.
Figure 3a.

Strap vicryl suture.
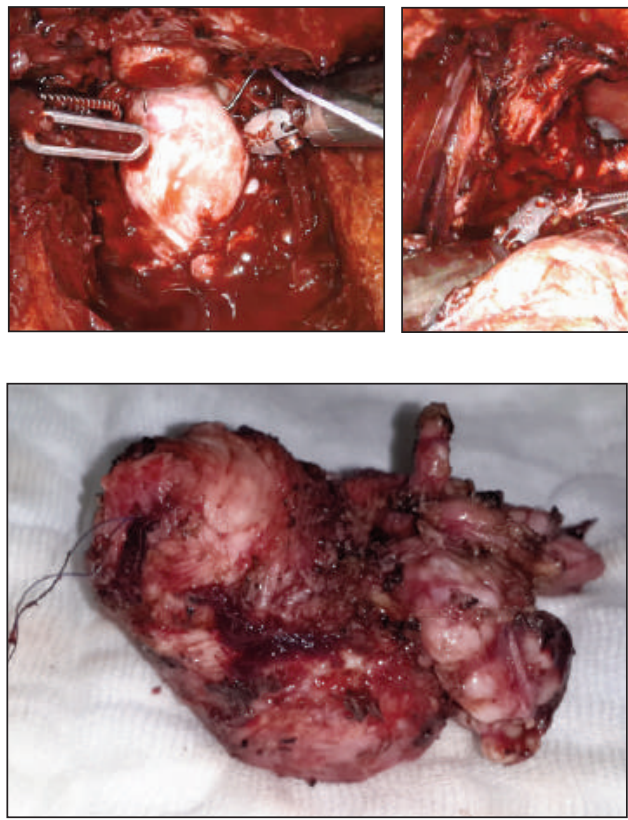

urethra from inside to outside clockwise down to 6 o'clock. A second barbed suture is used in the same setting but in reverse clockwise. Once the anastomosis is completed a $22 \mathrm{Ch}$ urethral catheter is replaced. The bladder is filled by $200 \mathrm{cc}$ saline to test the anastomosis for leakage. After observing the anastomosis is water tight, robotic system is undocked and a Jackson Pratt drain is placed. Specimen was sent to the pathology laboratory for examination (Figure 4).

\section{Discussion}

Today, despite the large prostate volumes, in the experienced centers, radical prostatectomy has been successfully applied with many techniques.

When we look at risk factors for prostate cancer, obesity and hypertension are at the forefront, therefore radical prostatectomy for large prostate volumes often is accompanied by obesity, hypertension and other comorbid factors. Consequently it is necessary to cope with the additional comorbidities and the negative factors related to previous abdominal surgery.

Sarle et al. reported that with retropubic approach the posterior border of the prostate base is very difficult to view, especially in patients with large median lobes, ureteral orifices are almost impossible to view and the risk of injury is high, especially in the antegrade approach, and seminal vesicle dissection prolonged the duration of the operation (5).

Despite the narrow surgical field with perineal approach, in our technique, large prostate was easily dissected with appropriate surgical margins on the apex, base and lateral planes, even if a large median lobe was present.

Approaching the posterior borders of the prostate from the inferior side allows to safely dissect them by using used strap vicryl suture without affecting the operation time with the ureters protected by direct vision. 
When large prostates are dissected from the bladder, large defects may form in the bladder neck and require additional reconstructive intervention to anastomose bladder neck to the urethra and can adversely affect continence (6). Eden et al. have proposed $60 \mathrm{cc}$ as the highest prostate volume for open perineal radical prostatectomy (7).

Regardless of how large the prostate, in our technique the anterior and posterior borders of the bladder can be easily visualized from perineal approach and the prostate can be easily dissected without creating large defects in the bladder neck.

Our technique allows to visualize the trigone and bilaterally the ureteral orifices. In patients who have large prostate, orifices can be protected by direct vision and bladder neck resection can be safely performed.

At the same time, the endopelvic fascia is completely preserved, the bladder neck defect is optimal for anastomosis and is not dissected from the surrounding tissues. All these advantages facilitate radical prostatectomy of large prostates in order to preserve the anatomic structure at the maximum extent and contribute to functional outcomes. This technique is safely applied to large prostates with maximum respect for anatomy with its advantages.

\section{Conclusion}

Robotic-PRP is a technique that can be applied safely without prolonging the operation period and without additional morbidity to the patient, preserving the oncologic and functional outcomes in patients with previous surgical history and large prostate volume.

\section{References}

1. Smith JA Jr, Chan RC, Chang SS, Herrell SD, et al. A comparison of the incidence and location of positive surgical margins in robotic assisted laparoscopic radical prostatectomy and open retropubic radical prostatectomy. J Urol. 2007; 178:2385-2389.

2. Hsu EI, Hong EK, Lepor H. Influence of body weight and prostate volume on intraoperative, perioperative, and postoperative outcomes after radical retropubic prostatectomy. Urology. 2003; 61:60-1.

3. Link BA, Nelson R, Josephson DY, et al. The impact of prostate gland weight in robot assisted laparoscopic radical prostatectomy. $J$ Urol. 2008; 180:928.

4. Tugcu V. Akca O, Simsek A, Yigitbasi I, et al. Robot-assisted radical perineal prostatectomy: first experience of 15 cases. Turk J Urol, 2017; 43:476-83.

5. Sarle R, Tewari A, Hemal AK, Menon M, Robotic-assisted anatomic radical prostatectomy: technical difficulties due to a large median lobe. Urol Int. 2005; 74:92-94.

6. Zorn KC, Orvieto MA, Mikhail AA, et al. Effect of prostate weight on operative and postoperative outcomes of robotic-assisted laparoscopic prostatectomy. Urology. 2007; 69:300-305.

7. Eden CG. Minimal access radical prostatectomy: how is it shaping up? BJU Int. 2008; 101:791-792.

\section{Correspondence}

Volkan Tugcu, MD

Abdulmuttalip Simsek, MD (Corresponding Author) simsek76@yahoo.com

İsmail Yigitbasi, $M D$

Mustafa Gürkan Yenice, MD

Selcuk Sahin, MD

Ali İhsan Tasci, MD

Bakirkoy Dr. Sadi Konuk Research and Training Hospital, Department of Urology Zuhuratbaba, Tevfik Saglam Cad. NO:11, Bakirkoy, Istanbul, Turkey 